\title{
EXPERIÊNCIAS COM O ANTIHELMÍNTICO BB-04 NO TRATAMENTO DE HELMINTOSES INTESTINAIS *
}

\author{
Léa Camillo_Coura, ** Adelina de S. Velho Soli, *** Hugo Americano Brasil **** \\ e J. Rodrigues Coura ******
}

\begin{abstract}
Os autores apresentam os resultados da associação piperazina-tiabendazoldimetilpolisiloxane no tratamento de infecções por helmintos intestinais. Os resütados vieram confirmar a apreciácel eficácia da piperazina e do tiabendazol pura o tratamento da ascaridíaso e da ancilostomiase nas doses utilizadas. Houve cura de $50 \%$ dos 82 casos de ancilostomiase, de $41 \%$ dos 72 ae ascaridiase $e$ de $26 \%$ dos 76 portadores de infecção pelo Trichuris trichiura.

Observou-se regressão da sintomatologia na maioria dos casos após instituída a terapêtica, especialmente dor abdominal, diarréia e obstipaçāo. A tolerância ao medicamento foi considerada boa.
\end{abstract}

E bastante conhecida a atividade da piperazina sôbre Ascaris lumbricoides e também sôbre o Enterobius vermicularis, sendo esta substância pràticamente incapaz de produzir, nas doses indicadas, manifestações de intolerância de importância, exceto em crianças epilépticas, pessoas debilitadas e em certas condições de hipersensibilidade à droga (3). O tiabendazol é também utilizado no arsenal terapêutico antihelmintico, especialmente para o tratamento da estrongiloidíase, sendo também apreciável a sua ação sôbre ancilostomídeos; efeitos colaterais são frequientemente relatados, especialmente quando administrado em dose única de $50 \mathrm{mg} / \mathrm{kg}(1,2$, $4,5)$.

O composto BB-04 contém hexahidrato de piperazina e tiabendazol, acrescidos da associação de dimetilpolisiloxane, silicone usado largamente como antiespumoso para combater o meteorismo e a flatulência, bem como a sensaçāo de mal-estar decorrente da dilatação gastrointestinal; sua associação à piperazina e ao tiabendozal em uma mesma preparaçāo farmacêutica foi feita numa tentativa de eliminar 0 mais ràpidamente os sintomas mencionados, freqüentemente encontrados em indivíduos poliparasitados.

O presente trabalho tem a finalidade de apresentar os resultados obtidos com êste preparado no tratamento de indivíduos parasitados por Ascaris lumbricoides, Trichuris trichiura e ancilostomídeos.

\section{MATERIAL E MÉTODOS}

Foram selecionados 82 pacientes dos Ambulatórios da Clínica de Doenças Infectuosas e Parasitárias da Faculdade de Medicina da U.F.R.J. e do Pôsto de Jacarepaguá da SUCAM, M. S.

* Trabalho da Clinica de Doenças Infectuosas e Parasitárias da Fac. de Med. da U.F.R.J. e do Instituto Brasileiro de Troplcologia Médica (Caixa Postal 1859), em colaboraçáo com a SUCAM, Mín. da Saúde.

* Professor assistente em tempo integral.

$\star * *$ Auxiliar de ensino.

$\star \star \star \star$ Chefe do Setor Jacarepaguá da Circunscriçăo Guanabara, SUCAM, M. S.

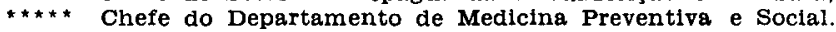


Quadro I

EXPERIENCIAS TERAPEUTICAS COM O VERMFFUGO BB-04

\begin{tabular}{|c|c|c|c|c|c|c|c|c|c|c|c|c|c|}
\hline \multirow{3}{*}{$\frac{\text { No }}{\text { ORDEM }}$} & \multirow{3}{*}{$\begin{array}{l}\text { INICIAIS } \\
\text { A. M. }\end{array}$} & \multirow{3}{*}{ IDADE } & \multirow{3}{*}{ SEXO } & \multirow{3}{*}{ COR } & \multicolumn{3}{|c|}{$\begin{array}{l}\text { EXAMES ANTERIORES } \\
\text { AO TRATAMENTO }\end{array}$} & \multirow{3}{*}{$\frac{\text { SINTOMAS PRE- }_{\text {TRATAMENTO }}}{\text { Dor abdominal }}$} & \multirow{3}{*}{ 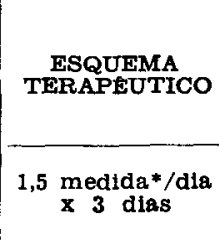 } & \multicolumn{4}{|c|}{$\begin{array}{l}\text { EXAMES APÓS } \\
\text { TRATAMENTO }\end{array}$} \\
\hline & & & & & Hoffman & \multicolumn{2}{|c|}{ Stoll } & & & \multicolumn{2}{|c|}{ Hoffman } & \multicolumn{2}{|c|}{ Stoll $* *$} \\
\hline & & & & & Anc Al $\mathbf{T t}$ & $\begin{array}{l}\text { Anc: } \\
\text { Al: } \\
\text { Tt: }\end{array}$ & $\begin{array}{r}200 \\
3000 \\
300\end{array}$ & & & Al & $T t$ & $\begin{array}{l}\text { Al: } \\
\text { Tt: }\end{array}$ & $\begin{array}{r}1200 \\
800\end{array}$ \\
\hline 2 & L. M. P. & $12 a$ & $\mathbf{M}$ & br & Anc Al $\mathrm{Tt}$ & $\begin{array}{l}\text { Anc: } \\
\text { Al: } \\
\text { Tt: }\end{array}$ & $\begin{array}{r}500 \\
10500 \\
200\end{array}$ & Dor abdominal & $1,5 \underset{x 3 \mathrm{dias}}{\operatorname{medida} / \mathrm{dla}}$ & Al 3 & $T t$ & $\begin{array}{l}\text { Al: } \\
\text { Tt: }\end{array}$ & $\begin{array}{l}1200 \\
2300\end{array}$ \\
\hline 3 & J. M. B. & $9 \mathrm{a}$ & $\mathbf{M}$ & br & Anc Al $\mathbf{T t}$ & $\begin{array}{l}\text { Anc: } \\
\text { Al: } \\
\text { Tt: }\end{array}$ & $\begin{array}{l}500 \\
900 \\
600\end{array}$ & $\begin{array}{l}\text { Obstipação; } \\
\text { dor abdominal }\end{array}$ & $1,5 \underset{x 3 \text { dias }}{\text { medida } / d i a}$ & Al & & Al: & 2600 \\
\hline 4 & E. U. C. & $13 a$ & $\mathbf{M}$ & pd & Anc Al $\mathrm{Tt}$ & $\begin{array}{l}\text { Anc: } \\
\text { Al: } \\
\text { Tt: }\end{array}$ & $\begin{array}{l}200 \\
300 \\
500\end{array}$ & - & 3 medidas/dia & Al & & Al: & 1500 \\
\hline 5 & J. L. Y. & $12 \mathrm{a}$ & $\mathbf{M}$ & br & Anc Al $\mathrm{Tt}$ & $\begin{array}{l}\text { Anc: } \\
\text { Al: } \\
\text { Tt: }\end{array}$ & $\begin{array}{l}900 \\
200 \\
500\end{array}$ & Dor abdominal & $\begin{array}{l}1,5 \text { medida/dia } \\
\times 3 \text { dias }\end{array}$ & Al 3 & & $\begin{array}{l}\text { Al: } \\
\text { Tt: }\end{array}$ & $\begin{array}{l}400 \\
150\end{array}$ \\
\hline 6 & J. H. $о$. & $6 \mathrm{a}$ & $\mathbf{M}$ & br & Anc Al Tt & $\begin{array}{l}\text { Anc: } \\
\text { Al: } \\
\text { Tt: }\end{array}$ & $\begin{array}{l}6000 \\
1000 \\
5000\end{array}$ & Dor abdominal & $1 \underset{x 3 \text { dias }}{\operatorname{medida} / d i a}$ & $T t$ & & Tt: & 150 \\
\hline$\eta$ & F. C. & $15 \mathrm{a}$ & $\mathbf{M}$ & br & Anc Al $\mathrm{Tt}$ & $\begin{array}{l}\text { Anc: } \\
\text { Al: } \\
\text { Tt: }\end{array}$ & $\begin{array}{r}10000 \\
6700 \\
3200\end{array}$ & Dor abdominal & 2 medidas/dia & neg. & & & \\
\hline 8 & E. $G$. & $12 a$ & $\mathbf{F}$ & br & Anc Al Tt & $\begin{array}{l}\text { Anc: } \\
\text { A1: } \\
\text { Tt: }\end{array}$ & $\begin{array}{r}2600 \\
1200 \\
700\end{array}$ & - & 3 medidas/dia & neg. & & & \\
\hline 9 & L. M. $O$. & $19 a$ & $\mathbf{F}$ & pd & Anc Al $\mathrm{Tt}$ & $\begin{array}{l}\text { Anc: } \\
\text { Al: } \\
\text { Tt: }\end{array}$ & $\begin{array}{r}300 \\
1800 \\
100\end{array}$ & $\begin{array}{l}\text { Fratulencia; obstipa- } \\
\text { çá; dor abdominal; } \\
\text { plrose }\end{array}$ & $\begin{array}{l}3 \text { medidas } / \mathrm{dia} \\
\mathrm{x} 3 \mathrm{dias}\end{array}$ & Anc & $\mathrm{Tt}$ & Anc: & $\begin{array}{l}150 \\
100\end{array}$ \\
\hline 10 & M. R. s. & $39 a$ & $\mathbf{F}$ & br & Anc $\mathrm{Al} \mathrm{Tt}$ & $\begin{array}{l}\text { Anc: } \\
\text { Al: } \\
\text { Tt: }\end{array}$ & $\begin{array}{r}10600 \\
6000 \\
600\end{array}$ & $\begin{array}{l}\text { Dlarréia; obstipaçáo; } \\
\text { dor abdomtnal; flatu- } \\
\text { lência; }\end{array}$ & $4 \underset{\times 3}{\text { medidas/dia }}$ & $\mathrm{Tt}$ & & $\mathrm{Tt}:$ & 400 \\
\hline 11 & M. A. F. & $13 a$ & $\mathbf{F}$ & pd & Anc AI Tt & $\begin{array}{l}\text { Anc: } \\
\text { Al: } \\
\text { Tt: }\end{array}$ & $\begin{array}{l}600 \\
900 \\
300\end{array}$ & Dor abdominal & 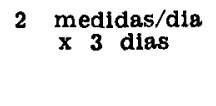 & Al & $\mathrm{Tt}$ & $\begin{array}{l}\text { Al: } \\
\text { Tt: }\end{array}$ & $\begin{array}{l}400 \\
150\end{array}$ \\
\hline
\end{tabular}

* Medtaa $=5$ ml. 
EXPERIENCIAS TERAPEUTICAS COM O VERMAFUGO BB-04 (Cont.)

\begin{tabular}{|c|c|c|c|c|c|c|c|c|c|c|c|c|}
\hline \multirow{3}{*}{$\frac{\begin{array}{c}\text { No } \\
\text { ORDEM }\end{array}}{12}$} & \multirow{3}{*}{$\frac{\text { INICIAIS }}{\text { C. A. S. }}$} & \multirow{3}{*}{$\frac{\text { IDADE }}{8 \mathrm{a}}$} & \multirow{3}{*}{$\frac{\text { SEXO }}{\mathrm{M}}$} & \multirow{3}{*}{$\frac{\text { COR }}{\text { pd }}$} & \multicolumn{3}{|c|}{$\begin{array}{c}\text { EXAMES ANTERIORES } \\
\text { AO TRATAMENTO }\end{array}$} & \multirow{3}{*}{$\begin{array}{l}\text { SINTOMAS PRE- } \\
\text { TRATAMENTO }\end{array}$} & \multirow{3}{*}{ 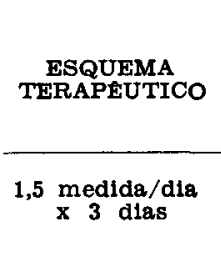 } & \multicolumn{3}{|c|}{$\begin{array}{l}\text { EXAMES APÓS } \\
\text { TRATAMENTO }\end{array}$} \\
\hline & & & & & Hoffman & \multicolumn{2}{|c|}{ Stoll } & & & \multirow{2}{*}{$\begin{array}{l}\text { Hoffman } \\
\text { Neg. }\end{array}$} & \multicolumn{2}{|c|}{ Stoll } \\
\hline & & & & & Anc $\mathrm{Al} \mathrm{Tt}$ & $\begin{array}{l}\text { Anc: } \\
\text { Al: } \\
\text { Tt: }\end{array}$ & $\begin{array}{r}300 \\
1800 \\
800\end{array}$ & & & & & \\
\hline 13 & s. C. & $5 a$ & $\mathbf{F}$ & $\mathrm{pd}$ & Anc Al Tt & $\begin{array}{l}\text { Anc: } \\
\text { Al: } \\
\text { Tt: }\end{array}$ & $\begin{array}{l}100 \\
300 \\
100\end{array}$ & Dor abdominal & $1 \underset{x 3 \text { dlas }}{\operatorname{medila} / \text { dia }}$ & Al & Al: & 250 \\
\hline 14 & J. P. T. & $38 a$ & $\mathbf{M}$ & br & Anc Al $\mathrm{Tt}$ & $\begin{array}{l}\text { Anc: } \\
\text { Al: } \\
\text { Tt: }\end{array}$ & $\begin{array}{l}100 \\
300 \\
100\end{array}$ & $\begin{array}{l}\text { Obstipaçá; dor abdo- } \\
\text { minal; flatulêncla; } \\
\text { pirose }\end{array}$ & 1 medida/dia & $\mathrm{Al}$ & Al: & 250 \\
\hline 15 & R. s. C. & $24 a$ & $\mathbf{M}$ & br & Anc $\mathrm{Al}$ Tt & $\begin{array}{l}\text { Anc: } \\
\text { AI: }\end{array}$ & $\begin{array}{r}800 \\
1500\end{array}$ & $\begin{array}{l}\text { Dlarréla; obstipaçáo; } \\
\text { dor abdominal; } \\
\text { flatulêncla }\end{array}$ & $4 \underset{x=3 \text { dias }}{\operatorname{medidas} / d i a}$ & - & $\mathrm{Tt}:$ & 50 \\
\hline 16 & J. E. s. & $52 a$ & $\mathbf{M}$ & $\mathrm{pd}$ & Anc Al Tt & $\begin{array}{l}\text { Anc: } \\
\text { AI: } \\
\mathrm{Tt}:\end{array}$ & $\begin{array}{r}2000 \\
6000 \\
500\end{array}$ & Flatuléncla & $4 \underset{x 3 \text { dias }}{\text { medidas } / d i a}$ & Al $\mathbf{T t}$ & $\begin{array}{l}\text { Al: } \\
\text { Tt: }\end{array}$ & $\begin{array}{r}500 \\
2700\end{array}$ \\
\hline 17 & M. S. & 53a & $\mathbf{F}$ & br & Anc Al Tt & $\begin{array}{l}\text { Anc: } \\
\text { Al: } \\
\text { Tt: }\end{array}$ & $\begin{array}{r}1200 \\
3000 \\
100\end{array}$ & $\begin{array}{l}\text { Dor abdominal; } \\
\text { flatuléncia }\end{array}$ & $4 \underset{x}{3}$ medias & Al $\mathrm{Tt}$ & $\begin{array}{l}\text { Al: } \\
\text { Tt: }\end{array}$ & $\begin{array}{l}300 \\
150\end{array}$ \\
\hline 18 & M. H. T. N. & $21 \mathrm{a}$ & $\mathbf{F}$ & br & Anc Al Tt & $\begin{array}{l}\text { Anc: } \\
\text { Al: } \\
\text { Tt: }\end{array}$ & $\begin{array}{l}1500 \\
1000 \\
2000\end{array}$ & $\begin{array}{l}\text { Dor abdominal; } \\
\text { flatulêncla; } \\
\text { pirose }\end{array}$ & 3 medidas/dia & Neg. & & \\
\hline 19 & M. A. & $20 \mathrm{a}$ & $\mathbf{F}$ & pd & Anc $\mathrm{Al} \mathrm{Tt}$ & $\begin{array}{l}\text { Anc: } \\
\text { Al: } \\
\text { Tt: }\end{array}$ & $\begin{array}{r}10000 \\
900 \\
2300\end{array}$ & Sem sintomatologia & $3 \underset{x 3 \text { dias }}{\text { medidas } / \text { la }}$ & $T t$ & Tt: & 450 \\
\hline 20 & v. C. A. & $9 a$ & $\mathbf{F}$ & pd & Anc Al $\mathbf{T t}$ & $\begin{array}{l}\text { Anc: } \\
\text { A1: } \\
\text { Tt: }\end{array}$ & $\begin{array}{r}400 \\
2600 \\
1100\end{array}$ & Dor abdominal & $\begin{array}{l}1,5 \text { medida } / \text { dia } \\
\times 3 \text { dias }\end{array}$ & Al $\mathrm{Tt}$ & $\begin{array}{l}\text { Al: } \\
\text { Tt: }\end{array}$ & $\begin{array}{l}300 \\
300\end{array}$ \\
\hline 21 & P. R. C. & $14 \Omega$ & $\mathbf{M}$ & pd & Anc Al Tt & $\begin{array}{l}\text { Anc: } \\
\text { Al: } \\
\text { Tt: }\end{array}$ & $\begin{array}{r}500 \\
900 \\
2500\end{array}$ & $\begin{array}{l}\text { Dlarré1a; dor } \\
\text { abdominal }\end{array}$ & $3 \underset{x 3 \text { dias }}{\operatorname{medtdas} / \text { dia }}$ & Al $\mathbf{T t}$ & $\begin{array}{l}\text { Al: } \\
\text { Tt: }\end{array}$ & $\begin{array}{l}150 \\
500\end{array}$ \\
\hline 22 & c. $\mathbf{s .}$ & $24 a$ & $\mathbf{F}$ & pd & Anc $\mathrm{Al} \quad \mathrm{Tt}$ & $\begin{array}{l}\text { Anc: } \\
\text { Al: } \\
\text { Tt: }\end{array}$ & $\begin{array}{l}200 \\
500 \\
100\end{array}$ & $\begin{array}{l}\text { Obstipaçăo; dor abdo- } \\
\text { minal; flatuléncla } \\
\text { pirose }\end{array}$ & $3 \underset{x 3 \text { dias }}{\operatorname{medtdas} / \text { dia }}$ & $\mathbf{T t}$ & Tt: & 350 \\
\hline
\end{tabular}


EXPERIENCIAS TERAPÊUTICAS COM O VERMAFUGO BB-04 (Cont.)

\begin{tabular}{|c|c|c|c|c|c|c|c|c|c|c|c|c|}
\hline \multirow{3}{*}{$\frac{\text { No }}{\text { ORDEM }}$} & \multirow{2}{*}{ INICIAIS } & \multirow{3}{*}{ IDADE } & \multirow{3}{*}{$\frac{\text { EEXO }}{\mathbf{F}}$} & \multirow{3}{*}{ COR } & \multicolumn{3}{|c|}{$\begin{array}{c}\text { EXAMES ANTERTORES } \\
\text { AO TRATAMENTO }\end{array}$} & \multirow{3}{*}{$\begin{array}{l}\text { SINTOMAS PRE- } \\
\text { TRATAMENTO }\end{array}$} & \multirow{2}{*}{$\begin{array}{c}\text { ESQUEMA } \\
\text { TERAPEUTICO }\end{array}$} & \multicolumn{3}{|c|}{$\begin{array}{l}\text { EXAMES APOS } \\
\text { TRATAMENTO }\end{array}$} \\
\hline & & & & & Hoffiman & \multicolumn{2}{|c|}{ Stoll } & & & Foffman & \multicolumn{2}{|c|}{ Stoll } \\
\hline & E. F. G. & & & & Anc Al Tt & $\begin{array}{l}\text { Anc: } \\
\text { Al: } \\
\text { Tt: }\end{array}$ & $\begin{array}{r}400 \\
3800 \\
8000\end{array}$ & & $1 \underset{\times 3 \text { dias }}{\text { medida/dia }}$ & $\begin{array}{l}\text { Anc Al } \\
\text { Tt }\end{array}$ & $\begin{array}{l}\text { Anc: } \\
\text { Al: } \\
\text { Tt: }\end{array}$ & $\begin{array}{r}100 \\
4900 \\
1750\end{array}$ \\
\hline 24 & A. P. $\mathbf{S}$. & 183 & $\mathbf{M}$ & br & A Ac Al Tt & $\begin{array}{l}\text { Anc: } \\
\text { Al: } \\
\text { Tt: }\end{array}$ & $\begin{array}{r}11000 \\
8500 \\
6000\end{array}$ & $\begin{array}{l}\text { Diarréia; dor abdomi- } \\
\text { nal; fratulência; } \\
\text { pirose }\end{array}$ & 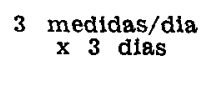 & Anc $T t$ & $\begin{array}{l}\text { Anc: } \\
\text { Tt: }\end{array}$ & $\begin{array}{l}1300 \\
1400\end{array}$ \\
\hline 25 & J. P. C. & $19 \mathbf{a}$ & $\mathbf{M}$ & $p d$ & Anc Al $\mathrm{Tt}$ & $\begin{array}{l}\text { Anc: } \\
\text { Al: } \\
\text { Tt: }\end{array}$ & $\begin{array}{l}7000 \\
8500 \\
5000\end{array}$ & $\begin{array}{l}\text { Dor abdominal; } \\
\text { flatulência; } \\
\text { plrose }\end{array}$ & $\begin{array}{l}4 \text { medidas } / \text { dia } \\
\times 3 \text { dias }\end{array}$ & Anc & Anc: & 2600 \\
\hline 26 & C. A. & $12 \mathrm{a}$ & $\mathbf{M}$ & pt & Anc Al $\mathrm{Tt}$ & $\begin{array}{l}\text { Anc: } \\
\text { Al: } \\
\text { Tt: }\end{array}$ & $\begin{array}{l}6000 \\
6000 \\
9000\end{array}$ & $\begin{array}{l}\text { Obstipaçäo; dor abdo- } \\
\text { minal; flatulência; } \\
\text { pirose }\end{array}$ & $\begin{array}{l}\text { 1,5 medida/dia } \\
\times 3 \text { dias }\end{array}$ & Al & Al: & 250 \\
\hline 27 & C. MI. J. V. & $9 \mathbf{a}$ & $\mathbf{M}$ & pd & Anc $\mathrm{Al} \mathrm{Tt}$ & $\begin{array}{l}\text { Anc: } \\
\text { Al: } \\
\text { T't: }\end{array}$ & $\begin{array}{l}9000 \\
7000 \\
3000\end{array}$ & $\begin{array}{l}\text { Obstipação; } \\
\text { dor abdominal }\end{array}$ & $\begin{array}{l}1,5 \text { medida/dia } \\
\times 3 \text { dias }\end{array}$ & Al $\mathrm{Tt}$ & $\begin{array}{l}\text { Al: } \\
\text { Tt: }\end{array}$ & $\begin{array}{l}300 \\
200\end{array}$ \\
\hline 28 & J. L. S. R. & $13 a$ & $\mathbf{M}$ & br & Anc Al $\mathrm{Tt}$ & $\begin{array}{l}\text { Anc: } \\
\text { Al: } \\
\text { Tt: }\end{array}$ & $\begin{array}{l}100 \\
400 \\
300\end{array}$ & Dor abdominal & $2 \underset{x 3 \text { dias }}{\operatorname{medidas} / \text { dia }}$ & $\underset{\mathrm{Tt}}{\operatorname{Anc}} \mathrm{Al}$ & $\begin{array}{l}\text { Anc: } \\
\text { Al: } \\
\text { Tt: }\end{array}$ & $\begin{array}{l}550 \\
100 \\
200\end{array}$ \\
\hline 29 & T. C. & ix & $\mathbf{F}$ & br & Anc Al $\mathrm{Tt}$ & $\begin{array}{l}\text { Anc: } \\
\text { Al: } \\
\text { Tt: }\end{array}$ & $\begin{array}{l}400 \\
900 \\
600\end{array}$ & Diarréia & 1 medida/dia & Anc $\mathrm{Tt}$ & $\begin{array}{l}\text { Anc: } \\
\text { Tt: }\end{array}$ & $\begin{array}{l}5500 \\
2250\end{array}$ \\
\hline 30 & V. F. P. & $6 a$ & $\mathbf{M}$ & br & Anc $\mathrm{Al} \mathrm{Tt}$ & $\begin{array}{l}\text { Anc: } \\
\text { Al: } \\
\text { Tt: }\end{array}$ & $\begin{array}{l}300 \\
600 \\
300\end{array}$ & Dor abdominal & 1 medida/dia & $\begin{array}{l}\text { Anc } \\
\text { Tt }\end{array}$ & $\begin{array}{l}\text { Anc: } \\
\text { Al: } \\
\text { Tt: }\end{array}$ & $\begin{array}{r}1500 \\
1200 \\
700\end{array}$ \\
\hline 31 & J. V. D. & $20 a$ & $\mathbf{M}$ & br & Anc $\mathrm{Al} \mathbf{T t}$ & $\begin{array}{l}\text { Anc: } \\
\text { Al: } \\
\text { Tt: }\end{array}$ & $\begin{array}{r}3000 \\
500 \\
1000\end{array}$ & $\begin{array}{l}\text { Obsttpação; dor abdo- } \\
\text { minal; flatulência; } \\
\text { pirose }\end{array}$ & $\begin{array}{l}4 \text { medidas } / \text { dia } \\
\times 3 \text { dias }\end{array}$ & Anc $T t$ & $\begin{array}{l}\text { Anc: } \\
\text { Tt: }\end{array}$ & $\begin{array}{l}450 \\
250\end{array}$ \\
\hline 22 & N. A. & $15 \mathrm{a}$ & $\mathbf{F}$ & pd & Anc Al Tt & $\begin{array}{l}\text { AI: } \\
\text { Ane: } \\
\text { Tt: }\end{array}$ & $\begin{array}{l}600 \\
500 \\
700\end{array}$ & $\longrightarrow$ & $\begin{array}{l}3 \text { medidas } / \text { dia } \\
\times 3 \text { dias }\end{array}$ & $\begin{array}{l}\text { Anc Al } \\
\text { Tt }\end{array}$ & $\begin{array}{l}\text { Anc. } \\
\text { Al: } \\
\text { Tt: }\end{array}$ & $\begin{array}{r}150 \\
50 \\
200\end{array}$ \\
\hline 33 & S. A. & $9 x$ & $\mathbf{F}$ & $\mathrm{pd}$ & Anc $\mathrm{Al} \mathrm{Tt}$ & $\begin{array}{l}\text { Anc: } \\
\text { Al: } \\
\text { Tt: }\end{array}$ & $\begin{array}{r}500 \\
1000 \\
500\end{array}$ & Do: abdominal & 1 medida/dia & $\underset{\mathbf{T t}}{\text { Anc }} \mathbf{A l}$ & $\begin{array}{l}\text { Anc: } \\
\text { Al: } \\
\text { Tt: }\end{array}$ & $\begin{array}{l}650 \\
\mathbf{5 5 0} \\
\mathbf{5 5 0}\end{array}$ \\
\hline
\end{tabular}




\begin{tabular}{|c|c|c|c|c|c|c|c|c|}
\hline \multirow{3}{*}{$\frac{\stackrel{\text { No }}{\text { ORDEM }}}{34}$} & \multirow{3}{*}{$\begin{array}{l}\text { INICIAIS } \\
\text { I. G. B. }\end{array}$} & \multirow{3}{*}{$\frac{\text { IDADE }}{25 a}$} & \multirow{3}{*}{$\frac{\text { SEXO }}{F}$} & \multirow{3}{*}{ COR } & \multicolumn{3}{|c|}{$\begin{array}{l}\text { EXAMES ANTERIORES } \\
\text { TRATAMENTO }\end{array}$} & \multirow{2}{*}{$\begin{array}{l}\text { SINTOMAS PRE- } \\
\text { TRATAMENTO }\end{array}$} \\
\hline & & & & & Hoffman & \multicolumn{2}{|c|}{ Stoll } & \\
\hline & & & & & Anc $\mathrm{Al} \mathbf{T t}$ & $\begin{array}{l}\text { Anc: } \\
\text { Al: } \\
\text { Tt: }\end{array}$ & $\begin{array}{r}1000 \\
1500 \\
600\end{array}$ & $\begin{array}{l}\text { Diarréia; } \\
\text { obstipaçáo; } \\
\text { dor abdominal; } \\
\text { flatulêncla; } \\
\text { pirose }\end{array}$ \\
\hline 35 & A. A. A. & $23 a$ & $\mathbf{M}$ & br & Anc Al $\mathrm{Tt}$ & $\begin{array}{l}\text { Anc: } \\
\text { Al: } \\
\text { Tt: }\end{array}$ & $\begin{array}{r}800 \\
20000 \\
6000\end{array}$ & Sem sintomatologia \\
\hline 36 & N. A. M. & $11 a$ & $\mathbf{F}$ & $\mathrm{pd}$ & Anc $\mathrm{Al} \mathrm{Tt}$ & $\begin{array}{l}\text { Anc: } \\
\text { Al: } \\
\text { Tt: }\end{array}$ & $\begin{array}{r}4000 \\
3000 \\
600\end{array}$ & Dor abdominal \\
\hline 37 & M. F. S. & $9 a$ & $\mathbf{F}$ & br & Anc $\mathrm{Al}$ Tt & $\begin{array}{l}\text { Anc: } \\
\text { Al: } \\
\text { Tt: }\end{array}$ & $\begin{array}{l}600 \\
600 \\
300\end{array}$ & Dor abdominal \\
\hline 38 & A. A. & $61 \mathrm{a}$ & $\mathbf{M}$ & br & Anc $\mathrm{Al} \mathrm{Tt}$ & $\begin{array}{l}\text { Anc: } \\
\text { Al: } \\
\text { Tt: }\end{array}$ & $\begin{array}{r}200 \\
1000 \\
300\end{array}$ & Sem sintomatologia \\
\hline 39 & M. B. L. & $21 a$ & $\mathbf{M}$ & br & Anc Al $\mathrm{Tt}$ & $\begin{array}{l}\text { Anc: } \\
\text { Al: } \\
\text { Tt: }\end{array}$ & $\begin{array}{r}800 \\
5000 \\
200\end{array}$ & $\begin{array}{l}\text { Dor abdominal; } \\
\text { flatulêncla; } \\
\text { pirose }\end{array}$ \\
\hline 40 & A. L. & $60 \mathrm{a}$ & $\mathbf{M}$ & br & Anc $\mathrm{Al} \mathrm{Tt}$ & $\begin{array}{l}\text { Anc: } \\
\text { Al: } \\
\text { Tt: }\end{array}$ & $\begin{array}{l}600 \\
700 \\
700\end{array}$ & $\begin{array}{l}\text { Dor abdominal; } \\
\text { flatulêncla; } \\
\text { pirose }\end{array}$ \\
\hline 41 & o. $\mathbf{M}$. & $52 a$ & $\mathbf{F}$ & pd & Anc Al Tt & $\begin{array}{l}\text { Anc: } \\
\text { Al: } \\
\text { Tt: }\end{array}$ & $\begin{array}{r}2500 \\
6000 \\
800\end{array}$ & $\begin{array}{l}\text { Obstipação; } \\
\text { pirose }\end{array}$ \\
\hline 42 & F. P. S. & $28 a$ & $\mathbf{M}$ & $\mathrm{pd}$ & Anc Al $\mathrm{Tt}$ & $\begin{array}{l}\text { Anc: } \\
\text { Al: } \\
\text { Tt: }\end{array}$ & $\begin{array}{r}10000 \\
6000 \\
3500\end{array}$ & $\begin{array}{l}\text { Flatulência; } \\
\text { pirose; }\end{array}$ \\
\hline 43 & M. I. 0 . & $39 a$ & $\mathbf{F}$ & $\mathrm{pd}$ & Anc Al $\mathrm{Tt}$ & $\begin{array}{l}\text { Anc: } \\
\text { Al: } \\
\text { Tt: }\end{array}$ & $\begin{array}{l}500 \\
300 \\
700\end{array}$ & $\begin{array}{l}\text { Dor abdominal; } \\
\text { flatulêncla; } \\
\text { pirose }\end{array}$ \\
\hline $44^{\circ}$ & c. 0 . & $11 \mathrm{a}$ & $\mathbf{F}$ & pd & Anc Al Tt & $\begin{array}{l}\text { Anc: } \\
\text { Al: } \\
\text { Tt: }\end{array}$ & $\begin{array}{r}800 \\
1000 \\
300\end{array}$ & Dor abdominal \\
\hline
\end{tabular}




\begin{tabular}{|c|c|c|c|c|}
\hline \multirow{3}{*}{$\begin{array}{c}\text { ESQUEMA } \\
\text { TERAPEUTICO } \\
\begin{array}{c}\text { medidas/dia } \\
\times 3 \text { dias }\end{array}\end{array}$} & \multicolumn{3}{|c|}{$\begin{array}{l}\text { EXAMES APSS } \\
\text { TRATAMENTO }\end{array}$} & \multirow{2}{*}{$\begin{array}{l}\text { SINTOMAS APOS } \\
\text { TRATAMENTO }\end{array}$} \\
\hline & Hoifman & \multicolumn{2}{|c|}{ STOLI } & \\
\hline & Anc Al & $\begin{array}{l}\text { Anc: } \\
\text { A.1: }\end{array}$ & $\begin{array}{r}1300 \\
\mathbf{3 0 0}\end{array}$ & $\begin{array}{l}\text { Melhorou da sintomatolozia. } \\
\text { à exceçũo de pirose. }\end{array}$ \\
\hline $3 \underset{x 3 \text { dias }}{\operatorname{medidas} / \text { dia }}$ & $\begin{array}{l}\text { Anc } A 1 \\
\text { Tt }\end{array}$ & $\begin{array}{l}\text { A nc: } \\
\text { Al: } \\
\text { It: }\end{array}$ & $\begin{array}{r}100 \\
4500 \\
100\end{array}$ & $\ldots$ \\
\hline $2 \underset{x}{\operatorname{medidas} / d i a}$ & Anc $\mathrm{Tt}$ & $\begin{array}{l}\text { Anc: } \\
\text { Tt: }\end{array}$ & $\begin{array}{r}1650 \\
700\end{array}$ & $\begin{array}{l}\text { Melhorcu da dor abdaminal. } \\
\text { Eliminou } 8 \text { exemplares de } A \text {. }\end{array}$ \\
\hline $\begin{array}{l}1,5 \text { medidia/dia } \\
\times 3 \text { dias }\end{array}$ & $\begin{array}{l}\text { Anc Al } \\
\text { Tt }\end{array}$ & $\begin{array}{l}\text { Anc: } \\
\text { Al: } \\
\text { Tt: }\end{array}$ & $\begin{array}{r}3600 \\
800 \\
300\end{array}$ & - \\
\hline $3 \underset{x 3 \text { dias }}{\operatorname{medidas} / \text { dia }}$ & neg. & & & - \\
\hline $4 \underset{x 3 \text { dias }}{\operatorname{medidas} / d i a}$ & Anc & Anc: & 1050 & $\begin{array}{l}\text { Melhorou da sintomatolozia. } \\
\text { Eliminou } 4 \text { exemplares de Al. }\end{array}$ \\
\hline $4 \underset{x 3 \text { dias }}{\operatorname{medidas} / d i a}$ & Anc $\mathrm{Al}$ & $\begin{array}{l}\text { Anc: } \\
\text { Al: }\end{array}$ & $\begin{array}{r}1550 \\
400\end{array}$ & $\begin{array}{l}\text { Melhorou da sintomatolozia. } \\
\text { Eliminou } 7 \text { exemplares de Al. }\end{array}$ \\
\hline 3 medidas/dia & $\begin{array}{l}\text { Anc } \mathrm{Al} \\
\mathrm{Tt}\end{array}$ & $\begin{array}{l}\text { Anc: } \\
\text { A1: } \\
\text { Tt: }\end{array}$ & $\begin{array}{l}250 \\
400 \\
250\end{array}$ & Melhorou da prisão-de-vantre. \\
\hline $4 \underset{x 3 \text { dias }}{\text { medidas } / \text { dia }}$ & $\begin{array}{l}\text { Anc } A l \\
\text { Tt }\end{array}$ & $\begin{array}{l}\text { Anc: } \\
\text { A1: } \\
\text { Tt: }\end{array}$ & $\begin{array}{l}1000 \\
1150 \\
1100\end{array}$ & $\begin{array}{l}\text { Nắo melhorou da slintona- } \\
\text { tclogla. }\end{array}$ \\
\hline $3 \underset{x 3 \text { dlas }}{\text { medidas } / \text { dla }}$ & Anc $\mathbf{T} t$ & $\begin{array}{l}\text { Anc: } \\
\text { Tt: }\end{array}$ & $\begin{array}{l}250 \\
150\end{array}$ & $\begin{array}{l}\text { Melhorou da sintomatologia. } \\
\text { Elixinou } 3 \text { exemplares de Al. }\end{array}$ \\
\hline $2 \underset{x<3 \text { dias }}{\operatorname{medidas} / d i a}$ & Anc. Al & $\begin{array}{l}\text { Anc: } \\
\text { Al: }\end{array}$ & $\begin{array}{r}50 \\
300\end{array}$ & $\begin{array}{l}\text { Melhorcu da sintomatciogta. } \\
\text { Elimincu } 2 \text { exemplares ct: Al. }\end{array}$ \\
\hline
\end{tabular}




\section{EXPERIÊNCIAS TERAPÊUTICAS COM O VERMIFUGO BB-04 (Cont.)}

\begin{tabular}{|c|c|c|c|c|c|c|c|c|c|c|c|c|}
\hline \multirow{3}{*}{$\begin{array}{c}\begin{array}{c}\text { No } \\
\text { ORDEM }\end{array} \\
45\end{array}$} & \multirow{3}{*}{ INICIAIS } & \multirow{3}{*}{$\frac{\text { IDADE }}{12 \mathrm{a}}$} & \multirow{2}{*}{ SEXo } & \multirow{2}{*}{ COR } & \multicolumn{3}{|c|}{$\begin{array}{c}\text { EXAMES ANTERIORES } \\
\text { AO TRATAMENTO }\end{array}$} & \multirow{2}{*}{$\begin{array}{l}\text { SINTOMAS PRE- } \\
\text { TRATAMENTO }\end{array}$} & \multirow{2}{*}{$\begin{array}{l}\text { ESQUEMA } \\
\text { TERAPEUTICO }\end{array}$} & \multicolumn{3}{|c|}{$\begin{array}{l}\text { EXAMES APÓS } \\
\text { TRATAMENTO }\end{array}$} \\
\hline & & & & & Holfman & & & & & Hof & $f \operatorname{man}$ & Stoll \\
\hline & & & $F^{*}$ & $\mathrm{pd}$ & Ane Al $\mathrm{T} t$ & Anc: & 1900 & Dor abdominal & $\begin{array}{l}1,5 \text { medida } / \text { dia } \\
\times 3 \text { dias }\end{array}$ & Anc & $\mathrm{Tt}$ & $\begin{array}{l}\text { Anc: } \\
\mathrm{Tt}:\end{array}$ \\
\hline 46 & D. A. & $8 a$ & $F$ & $\mathrm{pd}$ & Anc Al Tt & $\begin{array}{l}\text { Anc: } \\
\text { Al: } \\
\text { Tt: }\end{array}$ & $\begin{array}{r}2700 \\
6400 \\
800\end{array}$ & Dor abdominal & $\begin{array}{l}1,5 \mathrm{medida} / \mathrm{dia} \\
\times 3 \mathrm{dias}\end{array}$ & Anc & $\mathrm{Tt}$ & $\begin{array}{l}\text { Anc: } \\
\text { Tt: }\end{array}$ \\
\hline 47 & L. B. & $15 a$ & $F$ & br & Anc Al $\mathrm{Tt}$ & $\begin{array}{l}\text { Anc: } \\
\text { Al: } \\
\text { Tt: }\end{array}$ & $\begin{array}{r}1400 \\
500 \\
1600\end{array}$ & $\begin{array}{l}\text { Dor abdominal; diar- } \\
\text { réia; flatulência }\end{array}$ & $2 \underset{x 3 \text { dias }}{\text { medidas } / \text { dia }}$ & Anc & $\mathrm{Tt}$ & $\begin{array}{l}\text { Anc: } \\
\text { Tt: }\end{array}$ \\
\hline 48 & O. L. D. & $19 a$ & $\mathbf{M}$ & br & Anc $\mathrm{Al} \mathrm{Tt}$ & $\begin{array}{l}\text { Ane: } \\
\text { Al: } \\
\text { Tt: }\end{array}$ & $\begin{array}{r}2500 \\
6000 \\
900\end{array}$ & $\begin{array}{l}\text { Dor abdominal; flatu- } \\
\text { lência; pirose }\end{array}$ & $\begin{array}{l}3 \text { medidas/dia } \\
\times 3 \text { dias }\end{array}$ & $\begin{array}{l}\text { Anc } \\
\text { Tt }\end{array}$ & $\mathrm{A} 1$ & $\begin{array}{l}\text { Anc: } \\
\text { Al: } \\
\text { Tt: }\end{array}$ \\
\hline 49 & A. P. C. & $59 a$ & $F$ & pd & Anc Al $\mathrm{Tt}$ & $\begin{array}{l}\text { Anc: } \\
\text { Al: } \\
\text { Tt: }\end{array}$ & $\begin{array}{r}2500 \\
8000 \\
700\end{array}$ & - & $\begin{array}{l}3 \text { medidas/dia } \\
\times 3 \text { dias }\end{array}$ & $\underset{\mathrm{Tt}}{\mathrm{Anc}}$ & $\mathrm{AI}$ & $\begin{array}{l}\text { Anc: } \\
\text { Al: } \\
\text { Tt: }\end{array}$ \\
\hline 50 & M. c. s. & $4 a$ & $F$ & br & Anc Al $\mathrm{Tt}$ & $\begin{array}{l}\text { Anc: } \\
\text { Al: } \\
\text { Tt: }\end{array}$ & $\begin{array}{r}900 \\
1200 \\
600\end{array}$ & $\begin{array}{l}\text { Diarréia; dor abdomi- } \\
\text { nal; flatulência }\end{array}$ & $\begin{array}{c}1 . \mathrm{medida} / \mathrm{dia} \\
\times 3 \text { dias } \\
\end{array}$ & Anc & $\mathrm{Tt}$ & $\begin{array}{l}\text { Anc: } \\
\text { Tt: }\end{array}$ \\
\hline 51 & E. L. O. & $6 a$ & $F$ & br & Anc Al $\mathrm{Tt}$ & $\begin{array}{l}\text { Anc: } \\
\text { Al: } \\
\text { Tt: }\end{array}$ & $\begin{array}{l}800 \\
600 \\
300\end{array}$ & Obstipação & $1 \underset{x 3 \text { dias }}{\operatorname{medida} / \text { dia }}$ & $\mathrm{Tt}$ & & $T t:$ \\
\hline 52 & G. o. F. & $5 a$ & $F$ & br & Anc Al $\mathbf{T t}$ & $\begin{array}{l}\text { Anc: } \\
\text { Al: } \\
\text { Tt: }\end{array}$ & $\begin{array}{l}700 \\
200 \\
500\end{array}$ & Obstipaçāo & $1 \underset{x 3 \text { dias }}{\text { medida dia }}$ & $\mathrm{Tt}$ & & $\mathrm{Tt}:$ \\
\hline 53 & E. C. S. & $40 \mathrm{a}$ & M & br & Anc $\mathrm{Al} \mathrm{Tt}$ & $\begin{array}{l}\text { Anc: } \\
\text { Al: } \\
\text { Tt: }\end{array}$ & $\begin{array}{r}900 \\
1200 \\
600\end{array}$ & Flatulência & 3 medidas/dia & Anc & $\mathrm{Tt}$ & $\begin{array}{l}\text { Anc: } \\
\text { Tt: }\end{array}$ \\
\hline 54 & J. $\mathbf{x}$. & $19 a$ & $\mathbf{M}$ & br & Anc $\mathrm{Al} \mathrm{Tt}$ & $\begin{array}{l}\text { Anc: } \\
\text { Al: } \\
\text { Tt: }\end{array}$ & $\begin{array}{r}300 \\
600 \\
1000\end{array}$ & Pirose; dor abdominal. & $\begin{array}{l}3 \text { medidas } / \text { dia } \\
\times 3 \text { dias }\end{array}$ & $\underset{\mathrm{Tt}}{\text { Anc. }}$ & $\mathrm{Al}$ & $\begin{array}{l}\text { Anc: } \\
\text { Al: } \\
\text { Tt: }\end{array}$ \\
\hline 55 & J. C. A. M. & $7 a$ & $\mathbf{M}$ & br & Anc AI $T t$ & $\begin{array}{l}\text { Anc: } \\
\text { Al: } \\
\text { Tt: }\end{array}$ & $\begin{array}{r}5000 \\
1000 \\
200\end{array}$ & Flatulência & 1 medida $/$ dia & $\begin{array}{l}\text { Anc } \\
\text { Tt }\end{array}$ & $\mathrm{AI}$ & $\begin{array}{l}\text { Anc: } \\
\text { Al: } \\
\text { Tt: }\end{array}$ \\
\hline
\end{tabular}


EXPERIENCIAS TERAPÊUTICAS COM O VERMÎFUGO BB-04 (Cont.)

\begin{tabular}{|c|c|c|c|c|c|c|c|c|c|c|c|c|}
\hline \multirow{3}{*}{$\frac{\substack{\text { No } \\
\text { ORDEM }}}{56}$} & \multirow{3}{*}{ INICIAIS } & \multirow{3}{*}{$\begin{array}{c}\text { IDADE } \\
33 a\end{array}$} & \multirow{3}{*}{ SEXO } & \multirow{3}{*}{$\frac{\text { COR }}{b r}$} & \multicolumn{3}{|c|}{$\begin{array}{l}\text { AO TRATAMENTO } \\
\text { EXAMES ANTERIORES }\end{array}$} & \multirow{2}{*}{$\begin{array}{l}\text { SINTOMAS PRÉ- } \\
\text { TRATAMENTO }\end{array}$} & \multirow{2}{*}{$\begin{array}{c}\text { ESQUEMA } \\
\text { TERAPÊUTICO }\end{array}$} & \multicolumn{3}{|c|}{$\begin{array}{l}\text { EXAMES APOS } \\
\text { TRATAMENTO }\end{array}$} \\
\hline & & & & & Hoffman & \multicolumn{2}{|c|}{ Stoll } & & & Hoffman & \multicolumn{2}{|c|}{ Stoll } \\
\hline & & & & & Anc Al & $\begin{array}{l}\text { Anc: } \\
\text { Al: }\end{array}$ & $\begin{array}{l}300 \\
600\end{array}$ & - & $\begin{array}{l}3 \text { medidas } / \text { dia } \\
\times 3 \text { dias }\end{array}$ & Anc & Anc: & 100 \\
\hline 57 & C. A. L. & $9 a$ & $F$ & $\mathrm{br}$ & Anc $\mathrm{Al} \mathrm{Tt}$ & $\begin{array}{l}\text { Anc: } \\
\text { Al: } \\
\text { Tt: }\end{array}$ & $\begin{array}{r}700 \\
1700 \\
500\end{array}$ & Dor abdominal & $\begin{array}{l}1,5 \text { medida } / \text { dia } \\
\times 3 \text { dias }\end{array}$ & $\mathrm{T} t$ & $\mathbf{T t}:$ & 200 \\
\hline 58 & C. C. & $6 a$ & $\mathbf{M}$ & br & Anc $\mathrm{Al} \mathrm{Tt}$ & $\begin{array}{l}\text { Anc: } \\
\text { Al: } \\
\text { Tt: }\end{array}$ & $\begin{array}{r}2000 \\
1600 \\
500\end{array}$ & - & $\begin{array}{l}1 \text { medida } / \text { dia } \\
\times 3 \text { dias }\end{array}$ & Neg. & & \\
\hline 59 & M. F. & $44 a$ & $\mathbf{M}$ & br & Anc $\mathrm{Tt}$ & $\begin{array}{l}\text { Anc: } \\
\text { Tt: }\end{array}$ & $\begin{array}{r}800 \\
9600\end{array}$ & $\begin{array}{l}\text { Flatulência, azia, dor } \\
\text { abdominal }\end{array}$ & $\begin{array}{l}4 \text { medidas } / \text { dia } \\
\times 3 \text { dias }\end{array}$ & Anc Al & $\begin{array}{l}\text { Anc: } \\
\text { Al: }\end{array}$ & $\begin{array}{l}100 \\
300\end{array}$ \\
\hline 60 & G. J. & $5 a$ & $\mathbf{M}$ & $\mathrm{br}$ & Anc $\mathrm{Al}$ & $\begin{array}{l}\text { Anc: } \\
\text { Al: }\end{array}$ & $\begin{array}{r}700 \\
1000\end{array}$ & $\begin{array}{l}\text { Prisão de ventre; dor } \\
\text { abdominal }\end{array}$ & $\begin{array}{l}1 / 2 \mathrm{medida} / \mathrm{dia} \\
\times 3 \text { dias }\end{array}$ & Al $\mathrm{Tt}$ & $\begin{array}{l}\text { Al: } \\
\mathrm{Tt}:\end{array}$ & $\begin{array}{l}550 \\
300\end{array}$ \\
\hline 61 & C. J. V. & $11 a$ & $\mathbf{M}$ & br & Anc $\mathrm{A} 1$ & $\begin{array}{l}\text { Anc: } \\
\text { Al: }\end{array}$ & $\begin{array}{r}600 \\
1200\end{array}$ & $\begin{array}{l}\text { Prisão de ventre; dor } \\
\text { abdominal }\end{array}$ & $\begin{array}{c}1,5 \text { medida/dia } \\
\times 3 \text { dias }\end{array}$ & $\mathrm{Al}$ & Al: & 450 \\
\hline 62 & L. $\mathbf{S}$. & $28 a$ & $\mathbf{F}$ & br & Anc $\mathrm{Al} \mathrm{Tt}$ & $\begin{array}{l}\text { Anc: } \\
\text { Al: } \\
\text { Tt: }\end{array}$ & $\begin{array}{r}1000 \\
800 \\
900\end{array}$ & $\begin{array}{l}\text { Dor abdominal, } \\
\text { diarréia, flatulência }\end{array}$ & $\begin{array}{l}3 \text { medidas / dia } \\
x 3 \text { dias }\end{array}$ & $\begin{array}{l}\text { Anc AI } \\
\text { Tt }\end{array}$ & $\begin{array}{l}\text { Anc: } \\
\text { Al: } \\
\text { Tt: }\end{array}$ & $\begin{array}{l}400 \\
800 \\
800\end{array}$ \\
\hline 63 & M. A. & $4 a$ & $\mathbf{M}$ & br & Anc $\mathrm{Al} T \mathrm{Tt}$ & $\begin{array}{l}\text { Ane: } \\
\text { Al: } \\
\text { Tt: }\end{array}$ & $\begin{array}{l}300 \\
700 \\
900\end{array}$ & $\begin{array}{l}\text { Diarréia; dor } \\
\text { abdominal }\end{array}$ & $\begin{array}{l}1_{2} \text { medida } / \text { dia } \\
\times 3 \text { dias }\end{array}$ & $\begin{array}{l}\text { Anc } \mathrm{Al} \\
\mathrm{Tt}\end{array}$ & $\begin{array}{l}\text { Anc: } \\
\text { Al: } \\
\text { Tt: }\end{array}$ & $\begin{array}{l}300 \\
400 \\
150\end{array}$ \\
\hline 64 & J. G. & $8 a$ & $\mathbf{M}$ & br & Anc $\mathrm{Al} \mathrm{Tt}$ & $\begin{array}{l}\text { Anc: } \\
\text { Al: } \\
\text { Tt: }\end{array}$ & $\begin{array}{r}1000 \\
3000 \\
900\end{array}$ & - & $\begin{array}{l}1 \text { medida } / \text { dia } \\
\times 3 \text { dias }\end{array}$ & Anc Al & $\begin{array}{l}\text { Anc: } \\
\text { Al: }\end{array}$ & $\begin{array}{r}650 \\
1150\end{array}$ \\
\hline 65 & A. G. C. & $10 a$ & $\mathbf{F}$ & pt & Anc $\mathrm{Al} \mathrm{Tt}$ & $\begin{array}{l}\text { Anc: } \\
\text { Al: } \\
\text { Tt: }\end{array}$ & $\begin{array}{r}200 \\
3000 \\
100\end{array}$ & Dor abdominal & $\begin{array}{l}3 \text { medidas } / \text { dia } \\
\times 3 \text { dias }\end{array}$ & Al Tt & $\begin{array}{l}\mathrm{Al}: \\
\mathrm{Tt}:\end{array}$ & $\begin{array}{l}100 \\
150\end{array}$ \\
\hline 66 & E. B. & $5 a$ & $F$ & pd & Anc $\mathrm{Al} \mathrm{Tt}$ & $\begin{array}{l}\text { Anc: } \\
\text { Al: } \\
\text { Tt: }\end{array}$ & $\begin{array}{r}800 \\
2700 \\
800\end{array}$ & $\begin{array}{l}\text { Diarréta; dor } \\
\text { abdominal }\end{array}$ & 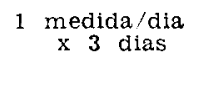 & Al $\mathrm{Tt}$ & $\begin{array}{l}\text { Al: } \\
\text { Tt: }\end{array}$ & $\begin{array}{l}1750 \\
1150\end{array}$ \\
\hline 67 & E. S. B. & $46 a$ & $\mathbf{F}$ & $\mathrm{br}$ & Anc $\mathrm{Al} T \mathrm{t}$ & $\begin{array}{l}\text { Anc: } \\
\text { Al: } \\
\text { Tt: }\end{array}$ & $\begin{array}{l}200 \\
100 \\
100\end{array}$ & Flatulência & $\begin{array}{l}3 \text { medidas } / \text { dia } \\
\times 3 \text { dias }\end{array}$ & A1 $\mathrm{Tt}$ & $\begin{array}{l}\text { Al: } \\
\text { Tt: }\end{array}$ & $\begin{array}{l}450 \\
450\end{array}$ \\
\hline 68 & L. G. & 7.8 & $\mathbf{F}$ & br & Anc $\mathrm{Al} \mathrm{Tt}$ & $\begin{array}{l}\text { Anc: } \\
\text { Al: } \\
\text { Tt: }\end{array}$ & $\begin{array}{l}500 \\
800 \\
600\end{array}$ & - & 1 medida/dia & $\begin{array}{l}\text { Anc Al } \\
\text { Tt }\end{array}$ & $\begin{array}{l}\text { Anc. } \\
\text { Al: } \\
\text { Tt: }\end{array}$ & $\begin{array}{r}950 \\
1000 \\
450\end{array}$ \\
\hline
\end{tabular}


EXPERIENCIAS TERAPEUTICAS COM O VERMIFUGO BB-04 (Cont.)

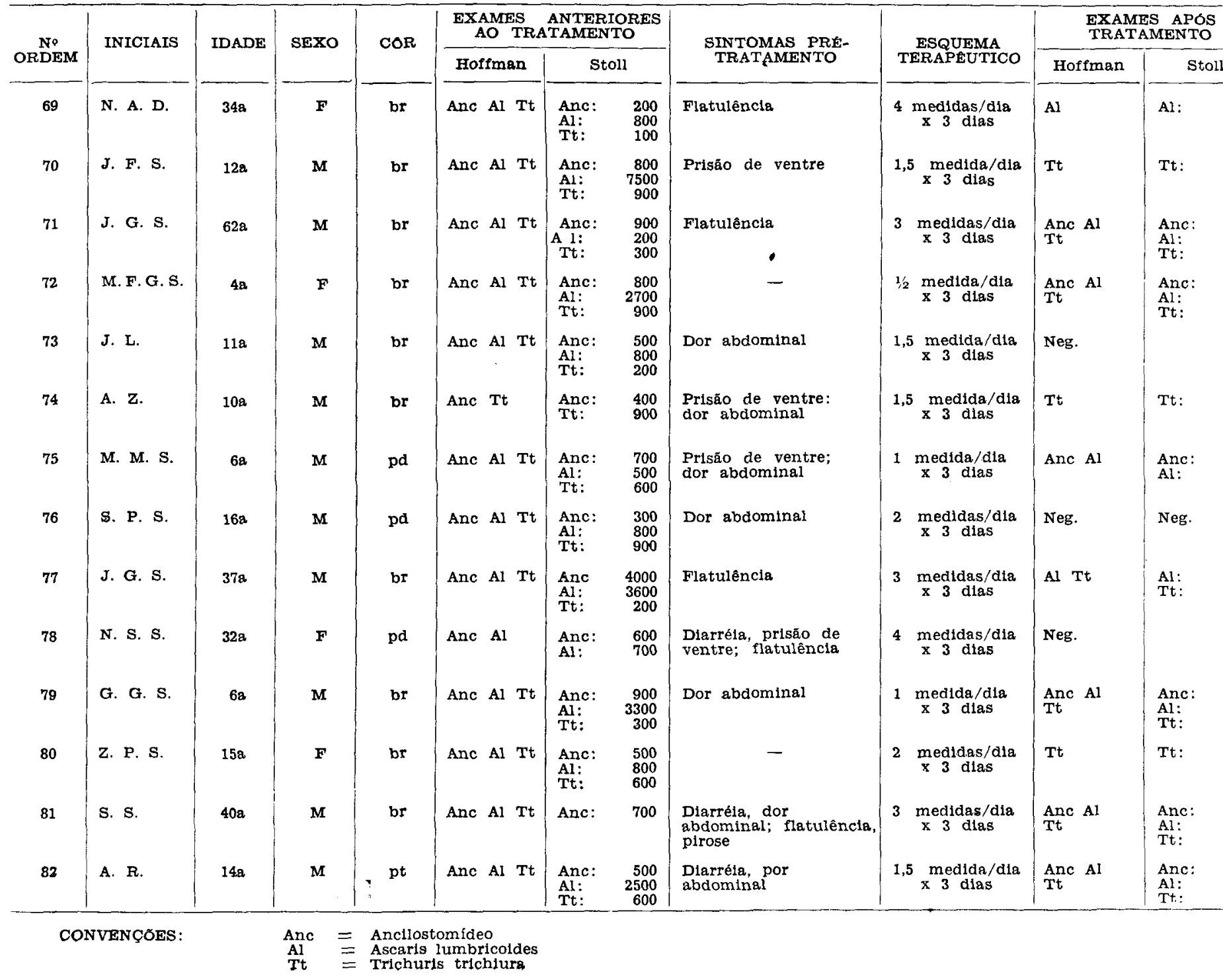


Os pacientes distribuiram-se, segundo os grupos etários, da seguinte maneira: 14 de 4 a 6 anos, 30 de 7 a 14 anos e 28 com mais de 14 anos, sendo as idades limite 4 e 62 anos; com relação à côr, 52 eram brancos, 27 pardos e 3 pretos. 45 eram do sexo masculino e 37 do feminino. (Quadro I) .

Encontravam-se parasitados por Ascaris lumbricoides 78 pacientes, por Trichuris trichiura 76 e por Ancylostomidae 82.

o diagnóstico coproscópico foi feito através da técnica de Hoffman-Pons Janner, complementada pela contagem pelo método de stoll; foram realizados no minimo dois exames de contrôle aos 7 e 15 a 20 dias após instituida a terapêtica, pelas mesmas técnicas já referidas. A maioria dos casos foi também submetida à coproscopia pela técnica de Willis.

Consideraram-se também, no contrôle de cura, as alterações apresentadas no que se refere à sintomatologia apresentada pelos pacientes.

O composto BB-04" é apresentado em solução.

Cada medida de $10 \mathrm{ml}$ contém $2,0 \mathrm{~g}$ de hexahidrato de piperazina (ou $1770 \mathrm{mg}$ de piperazine base), $500 \mathrm{mg}$ de tiabendazol e $42 \mathrm{mg}$ de metilpolisiloxane. O compasto foi administrado durante 3 dias consecutivos, à noite, ao deitar, em doses que va- riavam de acôrdo com o pêso, sendo administradas em cada tomada, doses aproximadas de $60 \mathrm{mg} / \mathrm{kg}$ de piperazina.

\section{RESULTADOS E COMENTÁRIOS}

Os quadros I e II nos permitem uma avaliação dos resultados obtidos com relação à cura parasitológica nos 82 pacientes tratados. Houve cura de $50 \%$ dos pacientes com ancilostomídeos, de $41 \%$ de infectados por Ascaris e de $26 \%$ de tricuríase. O percentual médio de redução do número de ovos por grama de fezes foi de $81 \%$ para ancilostomídeos, de $77 \%$ para Ascaris e de $67 \%$ para Trichuris trichiura.

No quadro III acha-se assinalada a sintomatologia antes $\mathrm{e}$ após instituída a terapêutica; 13 pacientes näo referiram quaisquer sintomas antes da terapêutica, permanecendo assintomáticos após a mesma; dor adbominal ocorria em 55, obstipação em 19, flatulência em 30 , pirose em 18 , diarréia em 14 casos, sendo que 35 pacientes apresentavam mais de uma queixa. Após o tratamento, não se evidenciaram melhoras em 18 dos 69 pacientes que referiram um ou mais dos sintomas relacionados; dêstes, 13 continuaram a apresentar fratulência; 12, dor abdominal; 6, obstipação; 10 , pirose e 2 , diarréia.

Observou-se eliminação de exemplares de Ascaris lumbricoides em 35 casos. Com

Quadro II

SINTESE DOS RESULTADOS OBTIDOS COM O VERMIFUGO BB-04

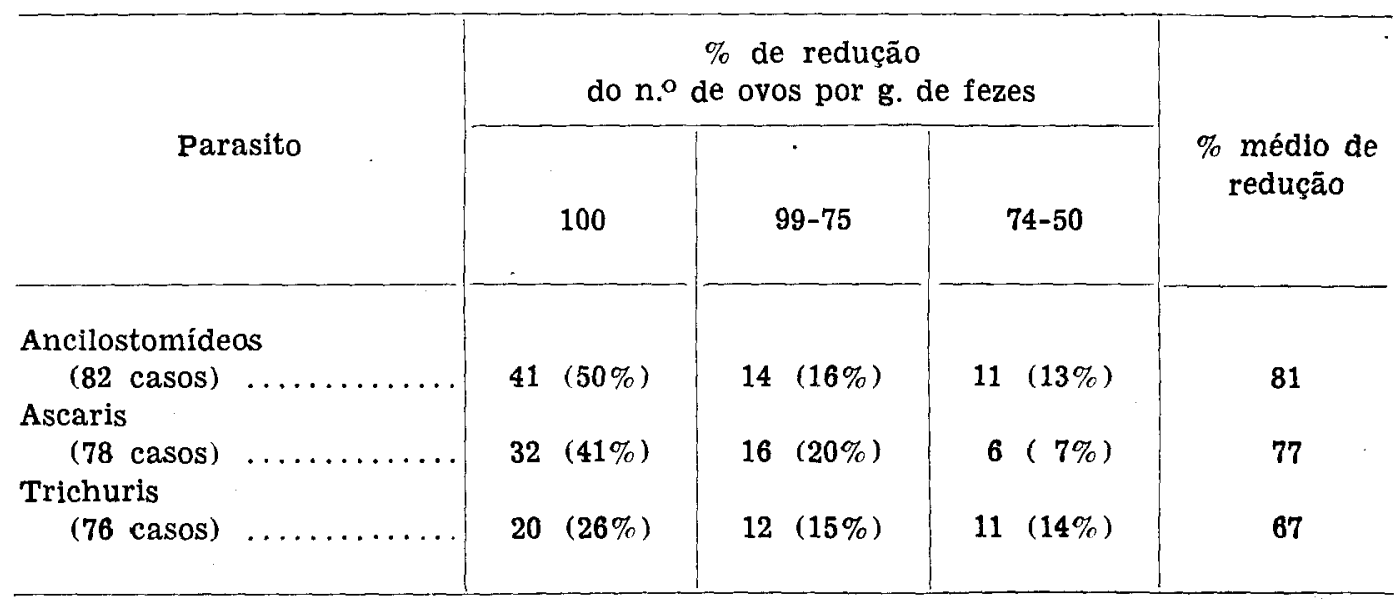

* Medicamento gentilmente fornecido pela Boehringer do Brasil S.A. 
Quadro III

SINTOMATOLOGIA APRESENTADA POR 82 PACIENTES ANTES E APÓS A TERAPEUTICA

\begin{tabular}{|c|c|c|c|c|}
\hline \multirow{2}{*}{ Sintoma } & \multicolumn{2}{|c|}{ Antes do tratamento } & \multicolumn{2}{|c|}{ Após o tratamento } \\
\hline & N. ${ }^{\circ}$ de casos & $\%$ & N. ${ }^{\circ}$ de casos & $\%$ \\
\hline Dor abdominal & 55 & 67,0 & 12 & 14,6 \\
\hline Flatulência $\ldots \ldots \ldots \ldots \ldots$ & 30 & 36,6 & 13 & 15,8 \\
\hline Obstipação & 19 & 23,1 & 6 & 7,3 \\
\hline Pirose $\ldots$ & 18 & 21,9 & 10 & 12,1 \\
\hline Diarréia & 14 & 17,0 & 2 & 2,4 \\
\hline Com associação de mais de & & & & \\
\hline um sintoma $\ldots \ldots \ldots \ldots$ & 35 & 42,6 & 9 & 10,9 \\
\hline $\begin{array}{l}\text { N. }{ }^{\circ} \text { total de casos com sin- } \\
\text { tomatologia } \ldots \ldots \ldots \ldots .\end{array}$ & & 81,7 & 18 & 21,9 \\
\hline
\end{tabular}

relação a manifestações de intolerância, um paciente relata ter tido náuseas com a primeira dose e um outro refere dor abdominal também com a primeira dose do Composto BB-04.

Os resultados obtidos no presente trabalho com relação à cura parasitológica são próximos aos referidos em outras investigações com a piperazina, nas doses empregadas, para tratamento da ascaridiase e com o tiabendazol, no tratamento da ancilostomíase; chama a atenção, no entanto, o percentual médio de reduçāo do número de ovos no caso da infecção pelo $T$. trichiura, superior aos habitualmente encontrados, embora o número de curas parasitológicas seja pouco significativo.
No que se refere à sintomatologia, houve melhoras em $74 \%$ dos casos, evidenciadas nos primeiros dias após instituida a terapêutica; êsses resultados parecem indicar, portanto, que, ao lado de cura parasitológica, observa-se também uma regressão considerável dos sintomas referidos pelos pacientes antes do tratamento, especialmente dor abdominal, diarréia e obstipação.

Considerando os resultados apresentados na presente casuística, e a boa tolerância à droga, acreditamos que esta associação medicamentosa possa ser utilizada para o tratamento de uma das associações parasitárias mais freqüentes $\mathrm{em}$ nosso meio, qual seja Ascaris lumbricoidesancilostomídeos.

\section{SUMMARY}

The authors present the results of the treatment of Ascaris, Trichuris and hookworm infection with an association of piperazine-thiabendazole and dimethilpolisiloxane; the cure rates were generally the achieved for ascariasis with dosis of piperazine of $60 \mathrm{mg} / \mathrm{kg}$ body weight for 3 days. $50 \%$ of the cases of hookworm infection cured after treatment; for whipworm infection the parasitological cure was very low - 26\% (of 76 patients). Tolerance was good. An improvement in clinical manifestations, specially abdominal pain, diarrhoea and obstipation was observed in most patients. 


\section{BIBLIOGRAFIA}

1) ALMEIDA JÚNIOR, N., REZENDE, $H$. P., BECKNER, Diva, BRANT, L. C., FERZEN, N., REIS, A. C. G., CARVALHAL, A. C., CASTILHO, A. M., LEAL, S. S., ERICKSEN, E. S., DIAMANTINO, J. E. \& TOSTES, R. O. G. - $O$ tratamento das infecçóes parasitárias múltiplas pelos derivados do tiabendazol. O Hospital, 76: 259, 1969 .

2) BOTERO, D. R. - Tiabendazol en el tratamiento de las helmintiasis intestinales. Antioquia Medica, 15: 565, 1965 .

3) CAMILLO-COURA, Léa \& CARVALHO, H. T. - Ascaridíase. Monografia. A Chimica Bayer, 1967.
4) RODRIGUES DA SILVA, J, CAMILLO-COURA, Léa, CARVALHo, H. T. \& LOPES, P. F. A. - $O$ tratamento da estrongiloidiase. Resultados preliminares de uma experiência com um nôvo antihelmíntico, o tiabendazol. O Hospital, 63: 1247, 1963.

5) VILELA, M. P., RODRIGUES, L. D., CAPELL, J. J., BRANDAOO, J. A., MARRIANI, I. \& ZUCATO, M. - $\mathrm{O}$ uso do tiabendazol no tratamento da estrongiloidíase e de outras infestações parasitárias no homem. O Hospital, 62: 691, 1962. 